\title{
一种复杂背景下红外目标稳定跟踪算法
}

\author{
李继泉, 时勤功, 胡春松 \\ (湖南华南光电（集团）有限责任公司, 湖南 常德 415000)
}

\begin{abstract}
摘要: 针对红外单目标在长期跟踪过程中的强背景干扰、遮挡、形变以及目标特征信息减弱等实际问 题, 提出了一种基于跟踪-学习-检测（Tracking-Learning-Detection, TLD）框架的红外目标稳定跟踪 方法。该方法在压缩跟踪算法 (Compressive Tracking, CT) 的基础上替换广义的类 Harr 特征为 HOG 特征, 引入互补随机测量矩阵, 优化纹理和灰度特征信息的权重, 同时引入卡尔曼滤波器记录空间上 下文位置信息, 以解决 CT 算法和 TLD 算法在目标被遮挡时的跟踪失效和全局检索问题。基于 TLD 算法框架和改进 CT 算法相结合的红外图像跟踪算法有效地解决了遮挡和强干扰问题，提升了算法的 跟踪准确性和长期跟踪稳定性。实验结果表明, 本文提出的算法在红外地面环境中能较好地实时稳定 跟踪并保持良好的准确性和鲁棒性。
\end{abstract}

关键词: 红外目标; 压缩跟踪算法; TLD 算法; HOG 特征; 卡尔曼滤波器

中图分类号：TP391.4 文献标识码：A 文章编号：1001-8891(2020)05-0434-06

\section{Stable Infrared Target Tracking Algorithm Under Complicated Background}

\author{
LI Jiquan, SHI Qingong, HU Chunsong \\ (Hunan Huanan Opto-Electro-Sci-Tech Co., LTD, Changde 415000, China)
}

\begin{abstract}
During the long-term tracking process of a single infrared target, many technical problems occur, such as strong background interference, occlusion, deformation, and target feature attenuation. An infrared target-tracking algorithm based on tracking-learning-detection (TLD) was proposed to solve these problems. Based on compressive tracking (CT), generalized Harr-like features were replaced by histograms of oriented gradient features. In our proposed method, a complementary random measurement matrix, which extracted texture and optimized grayscale feature-weights, was introduced. Moreover, a Kalman filter, used to record the space context location information, was adopted. Hence, the tracking failure and global retrieval problem of traditional CT and TLD algorithms can be solved when the target is occluded or deformed. The infrared image-tracking algorithm based on the combination of the TLD algorithm framework and improved CT algorithm effectively solves the problem of occlusion and strong interference and improves the tracking accuracy and long-term tracking stability of the algorithm. Experimental results show that the proposed algorithm can track well in real time and maintain good accuracy and robustness in an infrared ground environment.
\end{abstract}

Key words: compressive tracking, tracking-learning-detection, HOG features, Kalman filter

\section{0 引言}

红外成像技术, 作为衡量军事能力的技术之 一, 可适用于夜晚、雾霧、沙尘等能见度低的天气 情况, 具有全天候使用的特性。红外目标跟踪技术的 发展有利于军事武器系统地升级和现代化, 为此国 内外许多学者一直在不断地进行着深入研究。由于
复杂环境下的背景复杂, 红外图像信噪比较低, 目标 很容易被环境噪声淹没。因此, 如何在复杂背景下实 现红外目标实时、稳定地自主跟踪是当代红外技术 发展的关键问题之一。

目前经典的红外目标跟踪算法主要包括: 模板 匹配算法、光流跟踪算法以及均值漂移算法 ${ }^{[1]}$ 等。其 中, 模板匹配算法是一种较为简单有效的目标跟踪 
方法, 但是它存在着一个大的缺陷 ${ }^{[2]}$, 其目标跟踪匹 配的搜索区域会随着红外图像大小以及目标模板大 小的增加而急剧增大, 这会大大增加算法计算量并 降低目标跟踪的效率。光流跟踪算法是一种基于目 标运动的分析方法, 该算法在强噪声下, 跟踪不稳 定。均值漂移算法属于核密度估计法, 该算法对噪声 敏感, 易使算法不收玫。近年来, 研究人员基于压缩 感知思想 ${ }^{[3]}$, 提出了以压缩跟踪 (Compressive Tracking, CT) 为主的各种跟踪算法, 这些方法简单 高效, 但是对于复杂红外地面背景, 特征表现不够 稳定且对于形变和遮挡无法很好的处理。而 “跟踪学习-检测” (Tracking-Learning-Detection, TLD) 的 在线学习思想, 将目标跟踪算法与检测算法相结合, 能有效改善目标形变、目标部分被遮挡等问题。为 此, 我们提出了一种基于 TLD 算法框架的目标压缩 跟踪方法, 能够有效解决上述问题。

本文提出的跟踪算法能够改善 TLD 算法中值光 流的飘移以及 CT 算法的目标形变问题。本文着重研 究了以下 3 个主要问题: (1)在压缩跟踪特征提取过程 中引入互补测量矩阵, 以解决样本特征的不均匀问 题; (2)在压缩跟踪的特征累加计算中引入特征权 重, 以优化不同特征间的样本分类质量; (3)在经典 的 TLD 框架下融合改进的压缩跟踪算法, 改善 TLD 算法中值光流法的跟踪飘移问题以及压缩跟踪的目 标尺度变化问题。实验结果表明, 该红外目标跟踪 算法相对于传统红外目标跟踪算法, 在复杂红外地 面环境下, 提高了跟踪的准确性和鲁棒性。

\section{1 压缩跟踪算法和 TLD 算法}

\subsection{CT 算法简述}

CT 算法 ${ }^{[4]}$ 采用基于压缩感知理论提取压缩后的 特征, 其特征提取公式如下:

$$
\boldsymbol{v}=\boldsymbol{P} \boldsymbol{x}
$$

式中: $\boldsymbol{x} \in R(n \times l)$ 为样本原始特征矩阵; 矩阵 $\boldsymbol{P}(m \times$ $n)(m \ll n)$ 为满足约束等距性条件的随机测量矩阵; $\boldsymbol{v}(m$ $\times l)$ 为压缩后的特征。CT算法中随机测量矩阵 $\boldsymbol{P}$ 中元 素值的定义如下:

$$
r_{i, j}=\sqrt{c} \times \begin{cases}+1, & \text { 产生的概率为 } 1 /(2 c) \\ 0, & \text { 产生的概率为 } 1-1 / c \\ -1, & \text { 产生的概率为 } 1 /(2 c)\end{cases}
$$

式中: $r_{i, j}$ 为矩阵 $\boldsymbol{P}$ 中第 $i$ 行第 $j$ 列的元素; $c$ 的取值 随机在 2 3 2 之间 ${ }^{[4]}$ 选择。通过式(2)可以计算压缩特 征, 它是由原始特征以 $r_{i, j}$ 为权值的加权和, 其生成过 程如图 1 所示。

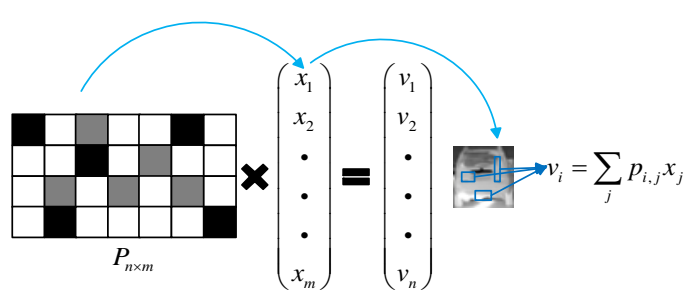

图 1 压缩特征 $\boldsymbol{v}$ 的生成示意图

Fig.1 Diagram of the generation of the compressed feature $\boldsymbol{v}$

CT 算法主要包括样本特征降维提取和朴素贝叶 斯分类器分类学习。其流程为: 先对目标区域进行特 征降维提取, 然后在目标区内提取正样本, 目标区外 提取负样本以训练 (更新) 贝叶斯分类器, 然后预测 目标位置。

\subsection{TLD 算法简述}

TLD 算法 ${ }^{[5]}$ 作为一种单目标长期跟踪算法, 其结 构如图 2 所示, 主要由检测、跟踪和学习 3 个模块构 成。其中, 检测模块和跟踪模块并行运行, 跟踪模 块应用光流法对目标位置进行估计，检测模块基于全 图使用分类器检测目标。学习模块基于跟踪器和检 测器的结果, 对跟踪模块的显著特征点进行更新, 同时更新检测器的模型。图 2 右侧的综合模块则结合 检测模块和跟踪模块结果融合输出。

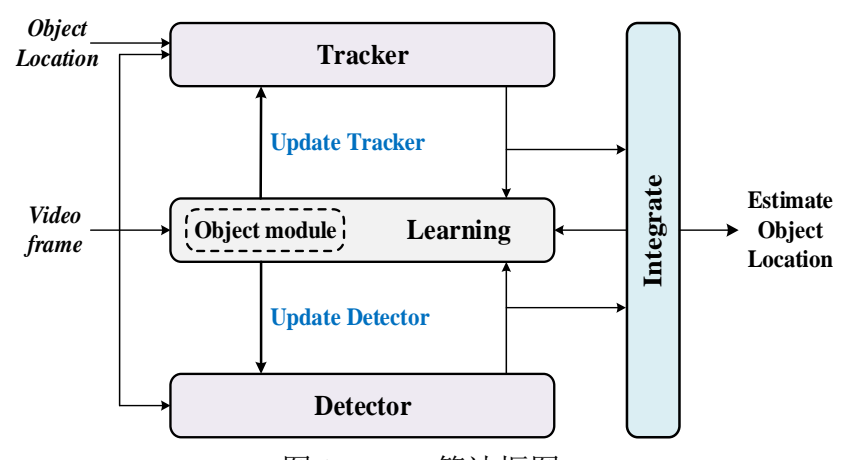

图 2 TLD 算法框图

Fig.2 The block diagram of TLD algorithm

\section{2 本文算法}

传统的 TLD 算法和 CT 算法都存在一定的不足, 其中 TLD 算法存在以下不足 ${ }^{[5]}$ : (1)目标被严重遮挡 时, TLD 检测器和跟踪器都无法捕获到目标, 从而 会造成目标丢失; (2)检测器全局检索目标时耗时且 计算量大； (3)基于目标直方图特征的光流法，易受 光照变化影响, 且在目标发生非刚性形变时, 光流法 跟踪易失效。

传统 CT 算法存在 Harr 特征在目标灰度剧烈变化 时表征不稳定，提取的纹理和灰度特征不均匀，以及 无法自适应目标尺度的变化。基于上述问题，我们提 出基于 TLD 算法框架的改进 CT 算法的红外图像跟踪 
算法。

\section{1 改进的压缩跟踪算法}

在跟踪算法中目标特征的提取和识别 ${ }^{[6]}$ 极其重 要, 而在复杂红外地面环境下, 背景杂波、运动模糊 和目标像素较少等问题, 均会对特征的提取和识别产 生影响。为解决上述问题, 首先使用局部光照不变性 和旋转平移不变性的 HOG 特征 $^{[7]}, h_{i}$ 来表征红外目 标, 以解决 Harr 特征的不稳定性 ${ }^{[4]}$ 。 HOG 特征其思 想是使用直方图来统计局部梯度方向的信息以表征 图像局部区域, 则特征提取变为式(3):

$$
v_{i}=\boldsymbol{P}_{i} h_{i}
$$

式中: $\boldsymbol{P}_{i}$ 为特征提取矩阵。在目标区域随机选择不同 尺度的矩形块 $T_{i}$ (2 $\sim 4$ 个), 统计每个 $T_{i}$ 的梯度方 向直方图 $h_{i}$, 以解决目标尺度变化问题。则特征提取 公式变为式(4), 其中 TN 为目标区域矩形块 $T_{i}$ 的总个 数:

$$
v_{i}^{k}=\sum_{k=1}^{T N} \frac{p_{i, k} h_{i, k}}{\sqrt{\mathrm{TN}}}
$$

红外目标特征主要构成为灰度和纹理特征, 且 灰度特征在目标运动时稳定, 纹理特征在环境和目 标纹理变化时稳定。且在低维特征向量空间下不同 特征对跟踪效果有较大的影响, 在低维特征向量空 间中， $62.5 \%$ 概率表征纹理特征，37.5\%概率表征灰 度特征 ${ }^{[8]}$ 。为了平衡纹理特征和灰度特征的提取对 稳定跟踪的影响, 本文引入原始测量矩阵的互补矩 阵。为了方便描述, 这里的系数随机测量矩阵用 $\boldsymbol{R}$ 表 示, 其互补随机测量矩阵用 $\boldsymbol{R}^{\prime}$ 表示, $\boldsymbol{R}^{\prime}$ 的元素参考 式(5):

$$
r_{i, j}^{\prime}= \begin{cases}(-1)^{j}\left|r_{i, j}\right| & \text { 第i行同号 } \\ \left|r_{i, j}\right| & \text { 第i行异号 }\end{cases}
$$

则特征提取模型变为: $v=\boldsymbol{R} x, v^{\prime}=\boldsymbol{R}^{\prime} x$ 。假定 $v$ 和 $v^{\prime}$ 中的特征元素相互之间独立分布, 则可对优化后的 特征使用朴素贝叶斯分类器建模, 且有先验概率 $p(y$ $=0)=p(y=1)$, 则由贝叶斯分类公式可得到式(6):

$$
\begin{aligned}
H(v)= & \lg \left(\frac{\prod_{i=1}^{m} p\left(v_{i} \mid y=1\right) p\left(v_{i}^{\prime} \mid y=1\right)}{\prod_{i=1}^{m} p\left(v_{i} \mid y=0\right) p\left(v_{i}^{\prime} \mid y=0\right)}\right)= \\
& \sum_{i=1}^{m} \lg \left(\frac{p\left(v_{i} \mid y=1\right) p\left(v_{i}^{\prime} \mid y=1\right)}{p\left(v_{i} \mid y=0\right) p\left(v_{i}^{\prime} \mid y=0\right)}\right)
\end{aligned}
$$

又由条件概率: $p\left(v_{i} \mid y=k\right)$ 符合正态分布, 即: $p\left(v_{i} \mid y=k\right) \sim N\left(\mu_{i}^{k}, \sigma_{i}^{k}\right), p\left(v_{i}^{\prime} \mid y=k\right) \sim N\left(\mu_{i}^{k}, \sigma_{i}^{k}\right)$ 。则 标量参数 $\mu_{i}^{y}, \sigma_{i}^{y}, \mu_{i}^{\prime y}, \sigma_{i}^{\prime y}$ 的更新公式通过式(7)可求:

$$
\left\{\begin{array}{l}
\mu_{i}^{y} \leftarrow \lambda \mu_{i}^{y}+(1-\lambda) \mu^{y}, \mu_{i}^{\prime y} \leftarrow \lambda \mu_{i}^{\prime y}+(1-\lambda) \mu^{\prime y} \\
\sigma_{i}^{y} \leftarrow \sqrt{\lambda\left(\sigma_{i}^{y}\right)^{2}+(1-\lambda)\left(\sigma_{i}^{y}\right)^{2}+\lambda(1-\lambda)\left(\sigma_{i}^{y}\right)^{2}} \\
\sigma_{i}^{\prime y} \leftarrow \sqrt{\lambda\left(\sigma_{i}^{\prime y}\right)^{2}+(1-\lambda)\left(\sigma_{i}^{\prime y}\right)^{2}+\lambda(1-\lambda)\left(\mu_{i}^{\prime y}-\mu^{\prime y}\right)^{2}}
\end{array}\right.
$$

式中: $\lambda(\lambda>0)$ 是学习速率参数; 参数 $\mu_{i}^{y}, \sigma_{i}^{y}, \mu_{i}^{\prime y}, \sigma_{i}^{\prime y}$ 由极大似然估计理论可得。

原始的朴素贝叶斯分类器将特征分类结果直接 相加, 易增大贝叶斯分类器误差, 造成目标模型漂 移。为了克服该问题, 我们对朴素贝叶斯分类器中 的特征进行加权处理, 式(8)给出了提取特征为目标 特征的概率:

$$
p_{i}(i)=\lg \left(p\left(v_{i} \mid y=1\right) / p\left(v_{i} \mid y=0\right)\right)
$$

这里引入特征权值 $w_{i}$ 来解决不同特征间的样本 分类 ${ }^{[9]}$ 。结合式(8)和阈值 $k$ 对分类器中特征 $v_{i}$ 进行评 估。如果 $p_{i}(i)>k$, 则样本分类结果为目标正样本; 反之为背景负样本。一般阈值 $k \geqslant 0$, 在此基础上更 新特征权值 $w_{i}$ 。由式 $w_{i}=\left(t p_{i}+f n_{i}\right) /\left(p_{i}+n_{i}\right)$ 可得权重 $w_{i}$ 。其中, $t p_{i}$ 表示正确分类的目标正样本个数, $f n_{i}$ 表示正确分类的背景负样本个数。则对于特征 $v_{i}$, 若 权值 $w_{i}$ 越大, 分类效果越好; 反之分类效果越差。故 贝叶斯分类器 $H_{\mathrm{n}}(v)$ 更新成式(9), 实际的跟踪器模板 为了更好的鲁棒性, 每 5 帧更新一次模型参数:

$$
H_{\mathrm{n}}(v)=\sum_{i=1}^{n} w_{i} \lg \left(\frac{\left(p\left(v_{i} \mid y=1\right) p\left(v_{i}^{\prime} \mid y=1\right)\right)}{\left(p\left(v_{i} \mid y=0\right) p\left(v_{i}^{\prime} \mid y=0\right)\right)}\right)
$$

\section{2 基于 TLD 框架整合优化 CT 算法}

基于 TLD 算法再检测机制实现的长期稳定跟踪 特性的框架。将改进的压缩跟踪算法整合到 TLD 框 架中, 提出改进的 CT (Improved Compressd Tracking, ICT）跟踪算法, 如图 3 所示。ICT 算法替代 TLD 算 法的跟踪模块, 能有效克服光流法红外跟踪带来的 漂移。同时 TLD 算法的在线学习机制, 能够提升红 外目标跟踪的稳定性, 也能弥补 ICT 算法的目标出视 野的问题, 有效地改善了算法的鲁棒性和准确性。

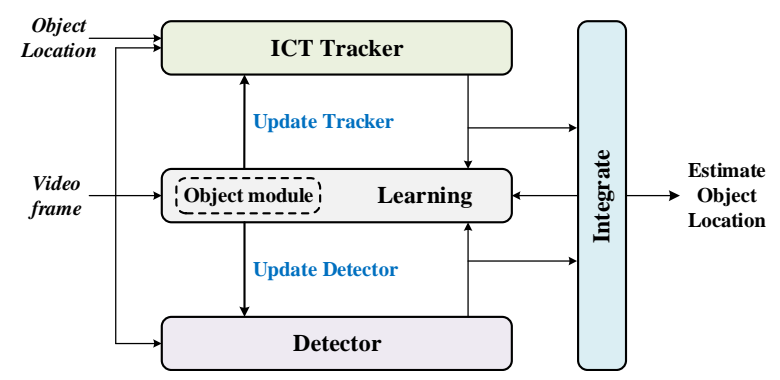

图 3 改进 TLD 算法与 ICT 算法融合的算法框架图

Fig.3 Improved method block diagram for fusion of TLD and ICT 


\section{3 遮挡目标预测}

目标跟踪算法在被遮挡后, 基于判别学习的方法 会学习到大量的负样本信息, 造成模型的漂移。CT 算法即使优化特征的抽取也无法有效地解决遮挡问 题。如果 ICT 跟踪模块跟踪的目标丢失, 且 TLD 的 检测模块无法在 2.5 倍目标区域内检测出目标, 此时 调用卡尔曼滤波器对目标接下来一帧的位置 Pos 进行 预测, 并在该位置的 2.5 倍目标区域面积大小区域内 进行检测。如果检测成功则重新初始化跟踪器和检测 器的参数, 否则执行 TLD 检测器进行全局检索。这 样在一定程度下有利于降低目标全局检索开销的时 间。

\section{4 本文算法实现}

在改进的 TLD 算法框架下结合 ICT 算法, 其实 现流程如图 4 所示。流程简述如下:

1) 第一帧初始化 ICT 算法的贝叶斯分类器、TLD 算法的随机森林分类器和 Kalman 滤波器参数; 2) 计 算测量矩阵 $\boldsymbol{R}$ 和 $\boldsymbol{R}^{\prime}$, 提取目标加权特征; 3) 计算 ICT 跟踪模块的响应结果 max_CT 和 TLD 检测器的响应 结果 max_DE, 更新 Kalman 滤波器位置参数, 并记 录目标位置和速度信息；4）根据阈值情况更新检测 模块和跟踪模块 (参考图 4 伪代码部分); 5) 循环 2) 4）步骤到视频结束。

\section{3 测试结果及分析}

基于自建视频测试集将改进算法与原算法 ( CT 算法、TLD 算法) 和红外主流稳定算法进行对比。对 比跟踪精度、跟踪抗遮挡、尺度变化等几个方面, 根据测试结果验证本文算法的有效性。

\section{1 视频序列}

视频序列采自国产非制冷红外成像系统, 主要 包含：行人、汽车等红外目标; 测试内容包含, 光 照变化, 目标遮挡、背景杂波、纹理变化、尺度变化 等。测试环境基于 Intel Core I5 台式计算机和 C ++ 语言。

\subsection{1 视频序列一}

实验一采用的是 $384 \times 288$ 分辨率的红外汽车视 频。跟踪结果如图 5 所示，其中跟踪框颜色指代不同 的算法。在跟踪前期阶段, 红外目标像素较少, 且和 背景灰度相近，参考第 10 帧结果， CT 算法出现轻微 的漂移, 其他两种算法稳定跟踪。在 26 帧以后, CT 算法目标漂移加剧, 后续的样本特征无法修正分类 器参数。再往后红外目标尺度变化明显, TLD 算法和 CT 算法均不能有效处理目标的尺度变化。本文算法 融合了两种算法的优点，同时在检测器中使用了多尺 度模板, 因而能够有效处理红外目标的尺度变化和 背景杂波干扰。
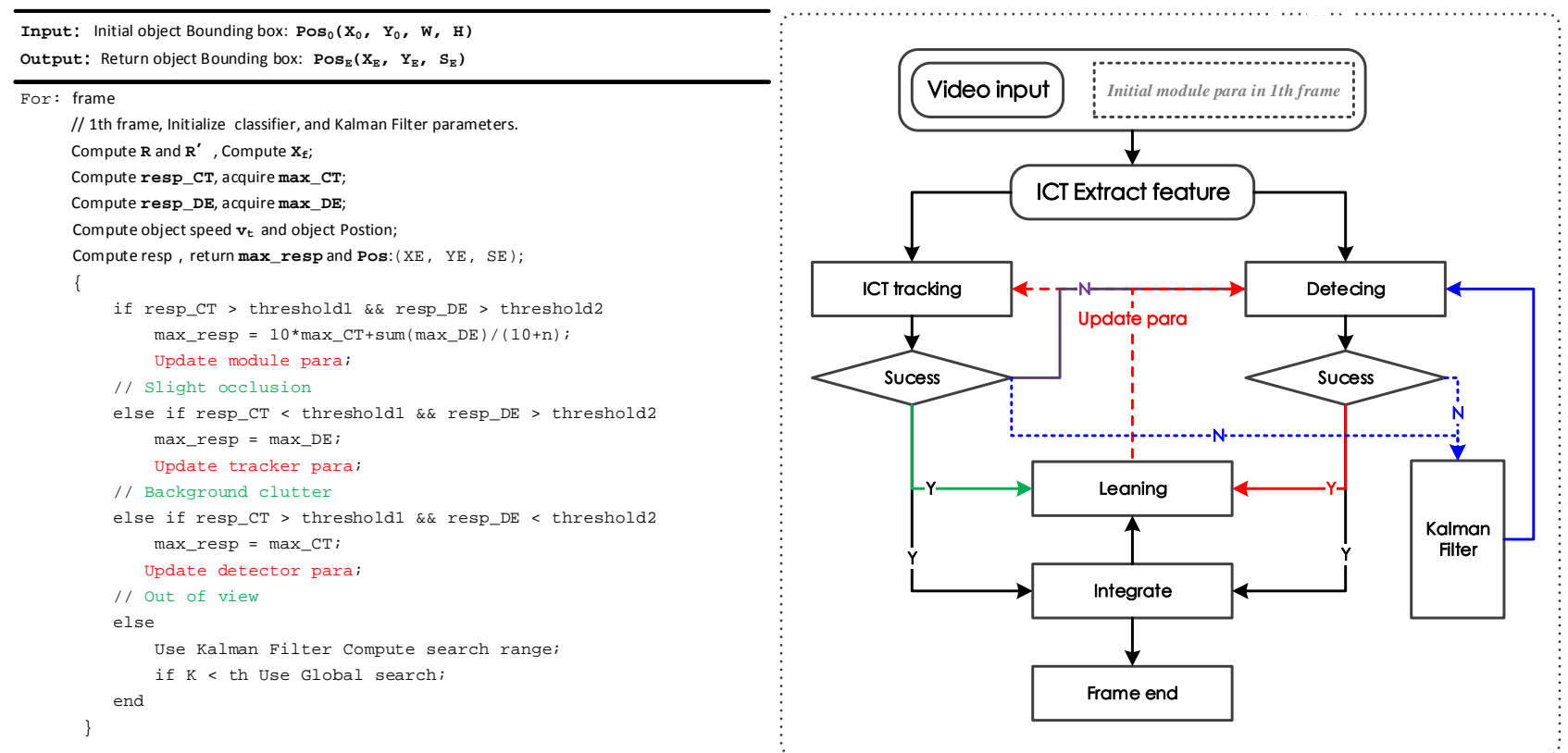

图 4 所提算法的伪代码和流程图

Fig.4 Pseudo code and flow chart of the proposed algorithm 

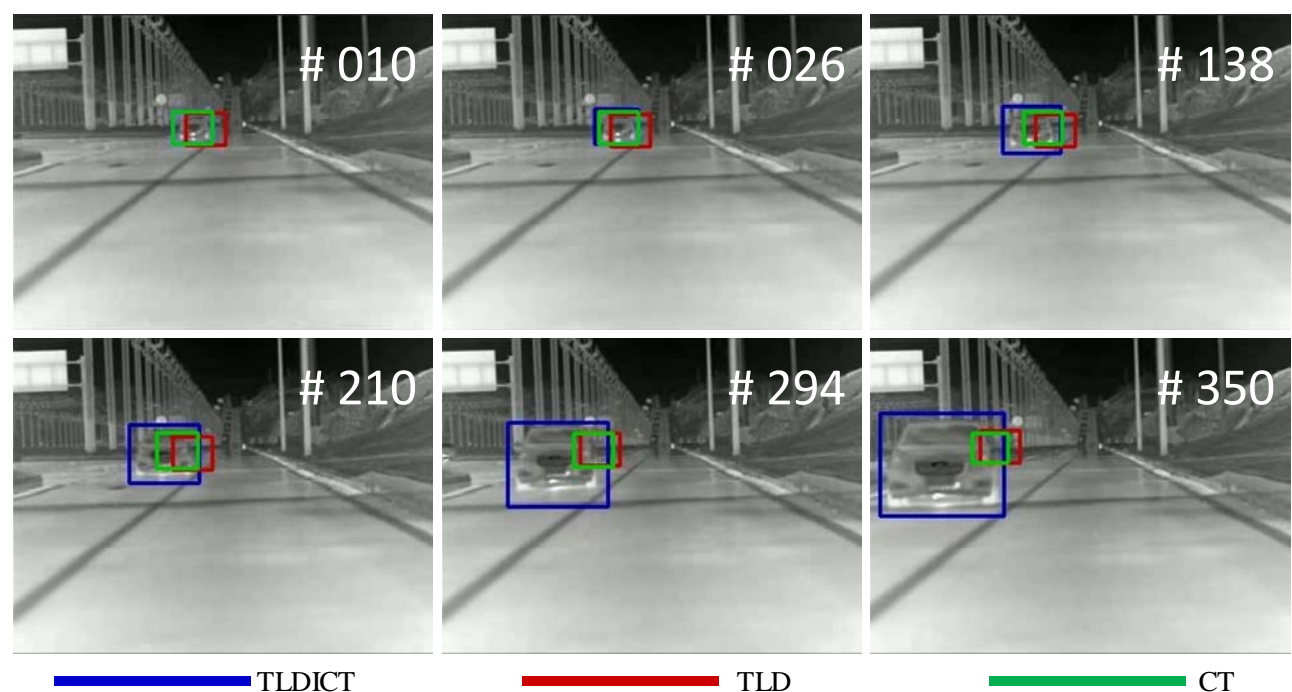

图 5 汽车红外视频的跟踪结果（第 10、26、138、210、294、350 帧）

Fig.5 Tracking results of car infrared video (10th, 26th, 138th, 210th, 294th, 350th frames)

再看上述情况的跟踪误差变化, 3 种算法跟踪误 差对比结果如图 6 所示。可以看出本文算法的总体跟 踪误差要明显优于压缩跟踪算法和 TLD 算法, 平均 误差在 3 个像素左右, 跟踪稳定。

\subsection{2 视频序列二}

实验二采用 $960 \times 576$ 分辨率的红外行人视频。 跟踪结果如图 7 所示, 其中跟踪框颜色指代不同的算 法。在跟踪前期阶段, 没有被遮挡或发生形变, 3 种 算法跟踪都较稳定。但在第 110 帧开始行人被树木遮 挡, TLD 算法由于负样本的学习导致模型漂移, 进而 目标框产生了漂移。CT 算法和本文算法对遮挡有较 强的鲁棒性。

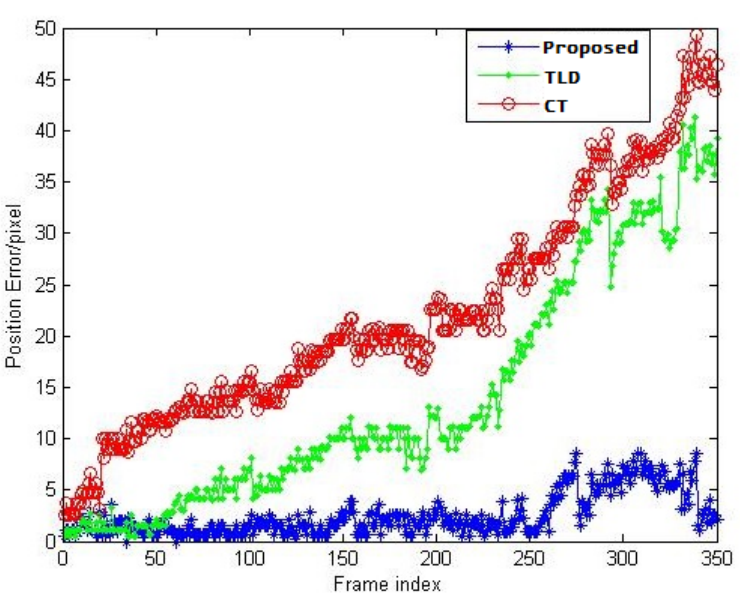

图 6 汽车红外视频的跟踪误差曲线图

Fig.6 Tracking error curves of car infrared video
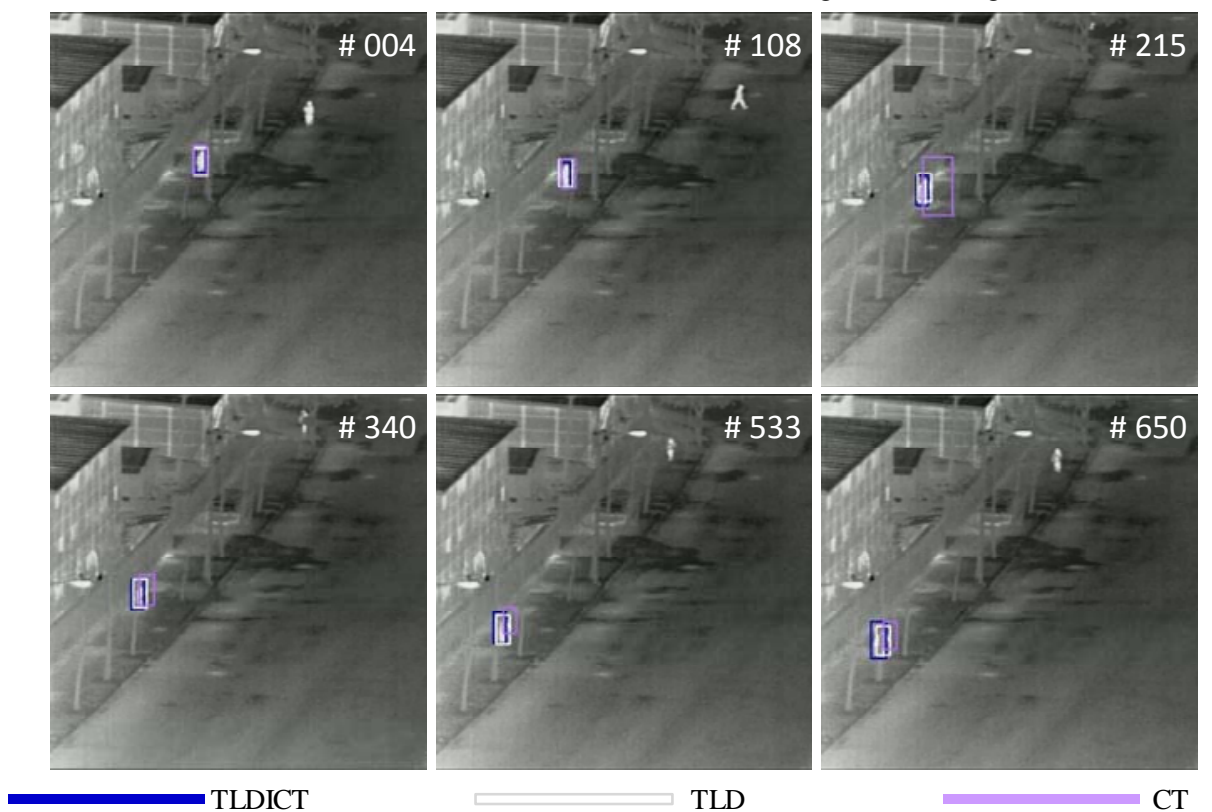

图 7 行人红外视频的跟踪结果（第 4、108、215、343、533、650 帧）

Fig.7 Tracking results of pedestrian infrared video (4th, 108th, 215th, 343th, 533th, 650th frames) 
在小目标不断被不同程度地遮挡变化情况下，3 种算法误差对比结果如图 8 所示。本文算法的总体跟 踪误差要明显优于 TLD 算法, 总体上可以看出, 本 文算法比 CT 算法的跟踪效果较好, 平均误差在 4 个 像素左右，跟踪效果良好。

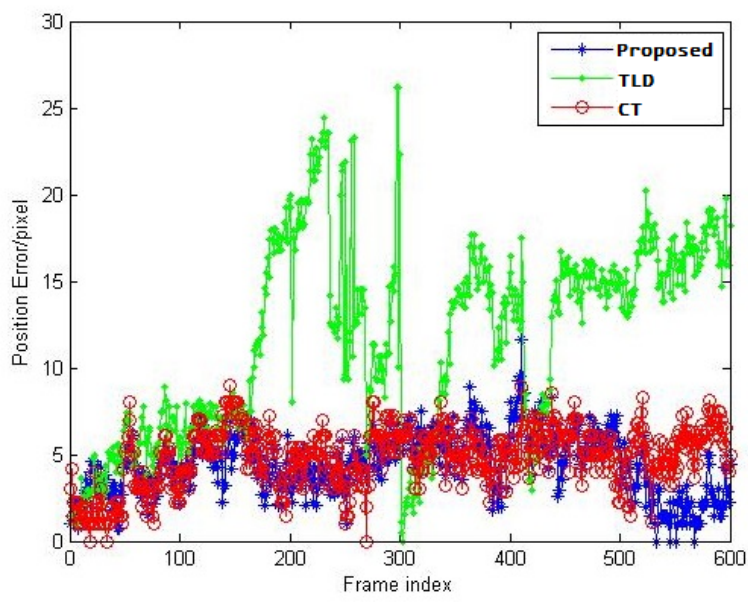

图 8 行人红外视频的跟踪误差曲线图

Fig.8 Tracking error curves of pedestrian infrared video

\section{2 对比其他算法}

通过 3.1 节可见提出的算法有效地解决了红外背 景杂波干扰、运动模糊、部分遮挡和目标轻微形变等 问题。再在 VOT-TIR2015 数据集对比测试经典的红 外目标跟踪算法, 如: $\mathrm{FCT}^{[9]}, \mathrm{IVT}^{[10]}, \mathrm{ODFS}^{[6]}$,

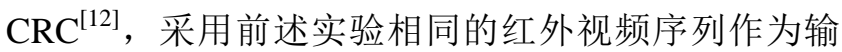
入。表 1 为各种跟踪算法的跟踪成功率和跟踪精度 ${ }^{[12]}$ 方面的对比情况, 其中跟踪成功率的两个衡量指标 为: Roc 曲线下的面积 (Area under Curve, AUC) 和 跟踪保持稳定的能力 (Track Maintenance, TM)。本 文算法在保证在前述平台下的 27FPS 速度还取得更 优异的性能。得益于改进 TLD 算法的在线学习和再 检测能力, 本文提出的跟踪算法在跟踪成功率和精 度方面相比其他算法都有了很大程度地提升, 同时保 证了较高的稳定性。

\section{表 1 几类红外常见跟踪算法的成功率和精度对比}

Table 1 Comparison of success rate and precision of several

\begin{tabular}{lccc}
\multicolumn{4}{c}{ common infrared tracking algorithms } \\
\cline { 2 - 3 } Methods & \multicolumn{2}{c}{ Success Rate } & Precision \\
\cline { 2 - 3 } & AUC & TM & \\
\hline FCT & 0.184 & 46.30 & 47.66 \\
IVT & 0.136 & 39.11 & 41.46 \\
ODFS & 0.125 & 36.00 & 38.64 \\
CRC & 0.118 & 33.54 & 34.22 \\
Proposed & 0.561 & 82.22 & 75.12 \\
\hline
\end{tabular}

\section{4 结语}

本文针对复杂地面背景下的红外目标稳定跟踪 问题提出了基于 TLD 框架的压缩跟踪方法, 该方法 将 CT 算法中表征不稳定的类 Harr 特征替换为 HOG 特征, 引入互补随机测量矩阵, 来自适应地平衡目标 纹理和灰度特征, 优化贝叶斯分类器的加权问题, 同时加入尺度处理模板, 很大程度上降低了传统红外 目标跟踪算法的模型漂移和遮挡。由于 TLD 框架的 在线学习机制, 有效地解决了目标脱离视野后的重补 问题, 如果出现遮挡使用卡尔曼滤波器进行区域预测 有效降低全局检测的复杂度, 这提升了跟踪算法的长 期准确性。融合 TLD 思想和 CT 算法的优点, 结合可 实时的速度, 有效地克服了红外目标跟踪地被遮挡和 形变等, 有利于目标地长期稳定跟踪。目前本算法只 是对单个目标进行跟踪, 如何将本文算法推广到多 目标和多种复杂条件下进行跟踪将是我们下一步将 要研究的重点方向。

\section{参考文献:}

[1] Comaniciu D, Ramesh V, Meer P. Real-time tracking of non-rigid objects using mean Shift[C]//EEE Computer Vision and Pattern Recognition, 2000: 142-149.

[2] YANG Yue, LIU Xingmiao, GUO Qiwang, et al. Infrared Object Matching Tracking Algorithm Based on Improved Mutual Information[J]. Infrared Technology, 2013, 35(6): 350-354.

[3] Elad M, Aharon M. Image denoising via sparse and redundant representations over learned dictionaries[J]. Image Processing, IEEE Transactions on, 2006, 15(12): 3736-3745.

[4] ZHANG K, ZHANG L, YANG M H. Real-time compressive tracking[C]//European Conference on Computer Vision, 2012: 864-877.

[5] Kalal Z, Mikolajczyk K, Matas J. Tracking-Learning-Detection[J]. IEEE Transactions on Pattern Analysis \& Machine Intelligence, 2012, 34(7): 1409-1422.

[6] ZHANG Kaihua, ZHANG Lei, YANG Mingsuan. Real-time object tracking via online discriminative feature selection[J]. Image Processing, IEEE Transactions on, 2013, 22(12): 4664-4677.

[7] Navneet Dalal, Bill Triggs. Histograms of oriented gradients for human detection[C]//IEEE Computer Vision and Pattern Recognition, 2005: 886-893.

[8] YANG H R. Research on tracking algorithm of infrared ground target [D]. Chengdu: University of Electronic Science and Technology of China, 2017.

[9] ZHANG Kaihua, ZHANG Lei, YANG Mingsuan. Fast compressive tracking Pattern Analysis and Machine Intelligence[J]. IEEE Transactions on, 2014, 36(10): 2002-2015.

[10] Ross D A, Lim J, Lin R, et al. Incremental Learning for Robust Visual Tracking[J]. International Journal of Computer Vision, 2008, 77(1): 125-141.

[11] Kalal Z, Matas J, Mikolajczyk K. P-N learning: Bootstrapping binary classifiers by structural constraints[C]//Computer Vision and Pattern Recognition, IEEE Xplore, 2010: 49-56.

[12] Felsberg Michael, Berg Amanda, Hager Gustav. The Thermal Infrared Visual Object Tracking VOT-TIR2015 Challenge Results[C]//2015 IEEE International Conference on Computer Vision Workshop (ICCVW), 2015: 639-651. 